\title{
Simulation of the Battery Pack Exploitation Based on Real Measurement Data of Applied Chemical Cells
}

\author{
Marcin Koniak, Andrzej Czerepicki, Piotr Tomczuk ${ }^{1}$, and Burak Şamşul ${ }^{2}$ \\ ${ }^{1}$ Warsaw University of Technology, Faculty of Transportation, 00-662, Koszykowa 75, Warsaw, Poland \\ ${ }^{2}$ Milper Propeller Technologies Inc., Teknopark Bulvarı - 1/2A Z08 34906, Pendik - İstanbul
}

\begin{abstract}
Authors of following article present the method of battery work simulation based on real measurement data of applied chemical cells and workload data. Description of method as its assumptions are presented. Next authors present workplace designed and constructed for acquiring operating characteristics according to assumed research plan. Article introduces computer system responsible for running simulations. At the end of publication results of selected runs are presented. Also described are types of tested cells and means of acquiring data concerning battery workload. Article ends with summarization and approximate plans of method development.
\end{abstract}

\section{Introduction}

Development of renewable energy sources and electric cars is inherent with use of batteries. Batteries applied in energetics have the task of storing energy that comes from low usage and overproduction from renewable sources and release it in consumption peak or during unfavourable weather, that causes lowered generation by wind turbines and solar panels. They can also function as a power peak check in nodes, breaking capacity and regulator of: frequency, voltage and power factor.

In case of electric vehicles battery is the only one, in other solutions main or as in hybrids supporting energy source. But in every single application its cost is substantial, and choice must be specifically made based on data closest to actual. Such choice is possible based on analysis of battery work in real operating conditions. In following article authors propose battery work simulation method based on characteristics of storage and actual measurement data used in chemical cells storage.

Described method was developed and utilized in international project "Renewable Energy Powered Hybrid Innovative Sailing Yacht" goal of which is development of yacht with electric engine powered from battery and characterized by possibility of charging from renewable source such as sun and wind.

\section{Method description}

There are many models describing battery work, among them: electric, electrochemical, analytical and stochastic. But a barrier for industrial application are simplifying assumptions and complexity of calculating procedure. Many of them require experimental data input, which can be only acquired with help of highly specialized lab tests.
Literature analysis shows, lack of universal methods that determine battery operating characteristics based on unified series of data for different technologies. Existing methods were not tested for newest technologies such as LFP, or lithium-titanate cells.

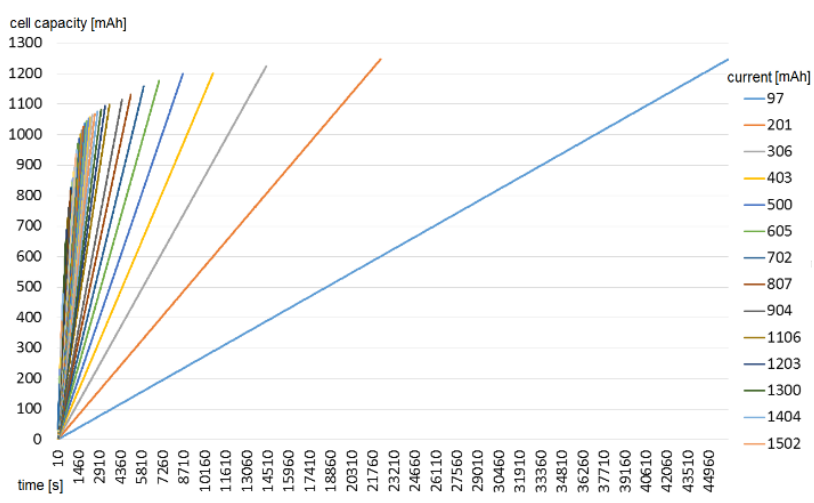

Figure 1. The dependence of available capacity, depending on the charging current as a function of the charging time.

The need for creation of a model projecting work of any battery was stated, for which input data can be acquired simply with help of universal research plan. In proposed solution fitting characteristic from a family of characteristics is plotted in response to instantaneous current of a cell, example of which can be found in Fig. 1. For each change in a current occurs a change of a characteristic that a process follows. Method assumes using real measurement data. So solution was developed and proposed that digitally models operating characteristic of a battery. Method assumes possibility of simulating whole battery by graduating a result of work simulation for a single cell. Possibility of such graduation was confirmed by a literature research [1], [2] and is a 
result of a previous experience team shares in that matter.

That angle takes into account such characteristics as:

- Available cell capacity related to charge/discharge current,

- Actual energy losses related to cell heating up,

- Change in a cell temperature related to operating current, this parameter is substantial for safety issues and allows to decide about addition of a cooling system to a battery.

For cell operation rating and proper selection of cell technology for a battery following data are essential:

- Operating temperature range,

- Allowed operating currents,

- Safety requirements,

- Service requirements,

- Assumed tray cost

- Influence of charging and discharging current on available capacity,

- Influence of temperature on available capacity,

- Correlation between lifetime and number and depth of ran work cycles [3].

Not all of the parameters above are available in manufacturers' materials. Only way of acquiring those missing data is running our own tests.

Running simulations of cells work would not be possible based only on manufacturers' catalogue data. So developing research plan was necessary, during which all necessary characteristics would be acquired. Such plan was made, also following criteria that must be met by measurements:

- Time constant of consecutive measurements for each cell must be as close as possible,

- Each measurement must consist of following data:

- Timestamp,

- Cell temperature,

- Work current,

- Energy sent/received,

- Cell voltage.

Key recorded parameters are described below:

\subsection{Current}

Operating current is a basic component influencing utilization of battery. Cell operating temperature, capacity and lifetime depend on its value. Because of that, range and step of measurements must be accordant to values in which cell will be eventually applied.

The first step is to find current from which measurements will start and it can be set at $0,1 \mathrm{~A}$.

Next, the step between consecutive measurements must be set and it can also be $0,1 \mathrm{~A}$.

Tests will be conducted until reaching: Maximal charging/discharging current permissible by manufacturer, or maximal assumed operating current in a pack for a given cell.

\subsection{Time}

Measuring time must be registered from a moment of its start until the end of the process. $5 \mathrm{~s}$ can be assumed as a time step between consecutive measurements. That value seems enough because changes occurring in cells have a slowly changing character. This parameter is necessary to control the progress of measurement data acquirement and duration of measurement, it also allows to verify completeness of collected results.

\subsection{Voltage}

Each cell must be tested only in the range given as safe in data provided by manufacturer. It will be confirmed in what range cell reaches capacity given by manufacturer and in that range it will be tested. Essential is also the way of voltage measurement, because measurement on the output of the device can be affected by error connected to voltage drop on power cords and all occurring connections eg. installed fuse or connectors.

\subsection{Number of work cycles}

Each type of cells will undergo tests that are intended to define actual number of work cycles. Those tests will consist of running series of charge/discharge measurements with given set operating currents, until reaching capacity loss equal to $30 \%$ of starting capacity. Those tests are planned for specified discharging depths, reflecting operating at full capacity, half and so called micro-cycles.

\subsection{Measurements results}

The measurements result for each cell is a catalog containing full measurement dataset acquired for each current. Tests will be run on workplace consisting of 4 independent measurement sets. Each of them will be running tests according to its own plan, adapted to operating parameters given by manufacturer. Tests monitoring will be possible from the computer.

\section{Research workplace}

It is necessary to run series of measurements in order to acquire data, that will be used in creation of individual cell types models. Those measurements must meet described earlier criteria.

Because of time-consuming research and necessary changes in operating currents in charging - discharging cycles, workplace must be controlled by computer with possibility of remote preview of results and operating conditions via Internet.

Two kinds of research are planned to take place at workplace:

- Accumulating work characteristics, which will become database for battery work simulation,

- Running series of charging and discharging measurements with specified operating currents, until reaching $30 \%$ loss of maximum capacity - cell lifetime tests.

Because of quality of measurement data it is necessary that environment in which cells are keeps constant temperature. Also temperature of cells must be constantly registered and monitored. In case of breaching 
assumed thresholds test must be cancelled, because of safety reasons.

Based on market analysis and specifications, integrated charger with specially modified for us firmware was selected as system that carries charging/discharging process. It can also be controlled via the serial port. It combines executive (programmable power supply, programmable workload) and measuring (currents, voltage, energy sent/received, temperature) systems. It assures processes to be executed in required range of currents and voltages for selected cells.

To ensure continuation of process in case of power blackout and to enable energy return from discharging cell, additional batteries are used in the power supply system. Each of four chargers is supplied from separate set: lab power supply and $12 \mathrm{~V}$ lead-acid battery as an energy buffer.

Keeping constant temperature of the process is electronically controlled refrigerator with Peltier cells.

With the controlling computer case is (as with chargers) keeping in mind supporting its work during blackouts, so laptop was selected because of built-in high capacity battery.

Finished measurement workplace is shown in Fig. 2. From the left: four programmable chargers, device keeping process temperature, controlling computer and batteries with power supplies.

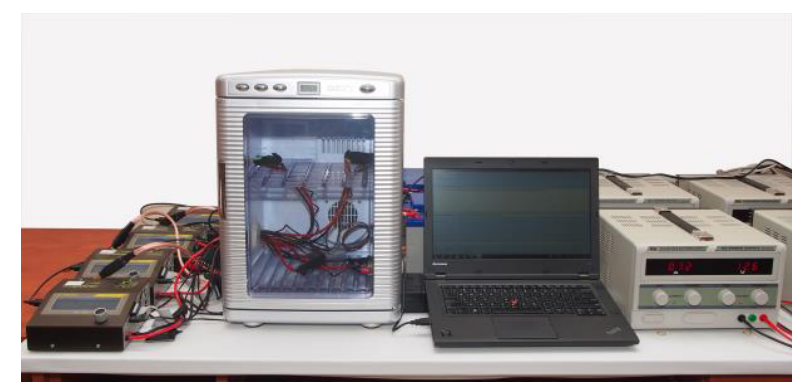

Figure 2. Laboratory test bench to collect the operating characteristics of chemical cells

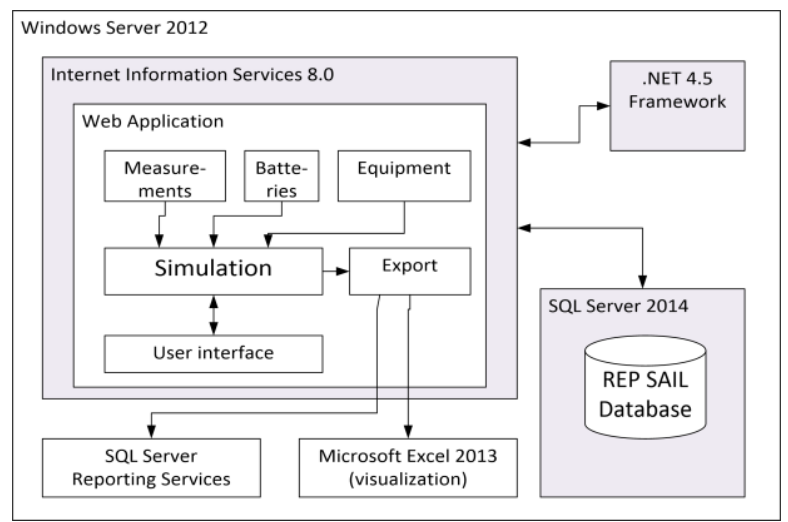

Figure 3. REP-SAIL IT system components

\section{Computer system}

REP SAIL computer system was designed with Microsoft technology. System platform is Microsoft Windows Server operating system. In order to store and process data relational database was used.
Application is available for user via WWW interface. Also there is provided possibility of sharing this application in Internet, what allows for remote access to research results for other members of consortium.

The WWW application is made of few interlocking modules: measurement supervision (import, validation, saving to database), battery supervision (defining properties and battery configuration), request supervision (defining workload and battery power supply), simulation of battery operation process in defined conditions, export to Excel to visualize data in table or pivot chart.

Microsoft Excel is optional component and can be installed on workstation to use data exported from system. Application is hosted on a WWW internet service server.

To run battery operation process simulation, simulation module input must contain following data:

- Configuration of battery for which simulation is run,

- Results of measurements for kind of cell on which battery is build,

- Data regarding energy flows (summed requirements for energy and delivered energy from power supplies),

- Starting battery charge level, in \% of maximum capacity.

All of the above should be saved in computer system database. Modelling algorithm involves an iterative calculation of battery energy changes according to formula 1 .

$$
E(t, I)=E_{0}+\sum_{n=1}^{k} \Delta E_{k}
$$

where:

$E_{0}$ - starting battery charge level,

$\Delta E_{k}$ - battery energy changes in time length of $\mathrm{t}\left(t_{i}, t_{i+1}\right)$, for which current has constant value, calculated as:

$$
\Delta E_{k}=E\left(t_{i+1}, I\left(t_{i+1}\right)\right)-E\left(t_{i}, I\left(t_{i}\right)\right)
$$

\section{Result examples}

For research in REP-SAIL project lithium technologies were selected because of their high volu- and gravimetric energy densities and possible high number of work cycles [4]. After market analysis following cells were selected:

- Li-ion: Panasonic NCR 18650B

- Li-ion: Samsung INR 18650-20r

- LiFePo4: A123 ANR 26650

- Li-poly: REDOX battery

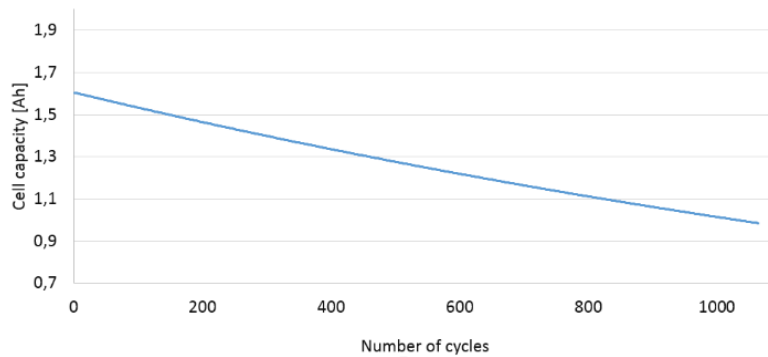

Figure 4. The dependence of the available capacity versus the number of cycles for the depth of discharge of $50 \%$ of total capacity. 
For them operating characteristics were acquired in current ranges permitted by cells manufacturers. Additionally they were tested for collected currents at cycling life time angle. Fig. 4 shows example aging characteristic based on the tests results.

This kind of tests allow to determine if aging process is linear or if at some moment characteristic breaks and there is need to include it at selection and exploitation of such battery [5].

To make operational characteristics for batteries in assumed applications it is necessary to acquire progress of workload with which they will be loaded. There are many ways of acquiring that type of data, including:

- Measuring real objects. It requires dedicated gear and service time. Results are most accurate and their research value - highest.

- Simulations using existing work models based on physical dependences. In this case results require additional validation. Acquired progress may differ from actual values, the cause - simplifications occurring in used models.

- Simulations using real measurement data. Example can be characteristics of bus ride time based on schedule and traffic modelled with help of actual operational runs. That way of simulation is flexible and can be used in scenarios in which real measures are impossible. Acquired results satisfactory reflect real conditions.

- Demand prognosing with help of summing power of receivers used in vehicle in function of time and assumed drive operating characteristic. This method is inaccurate, but allows for easy calculation of heaviest possible exploitation scenarios.

Created simulation method of battery work is excellent in cooperation with any of the mentioned above models. In energetic applications most popular data sources are measurements acquired in actual objects. Example set of data is shown in Fig. 5. This characteristic consists of measurements of such devices as: fridge, basic load, washing machine, dishwasher, kettle and household solar power plant. The total load current is converted to one that corresponds to the work of single cell.

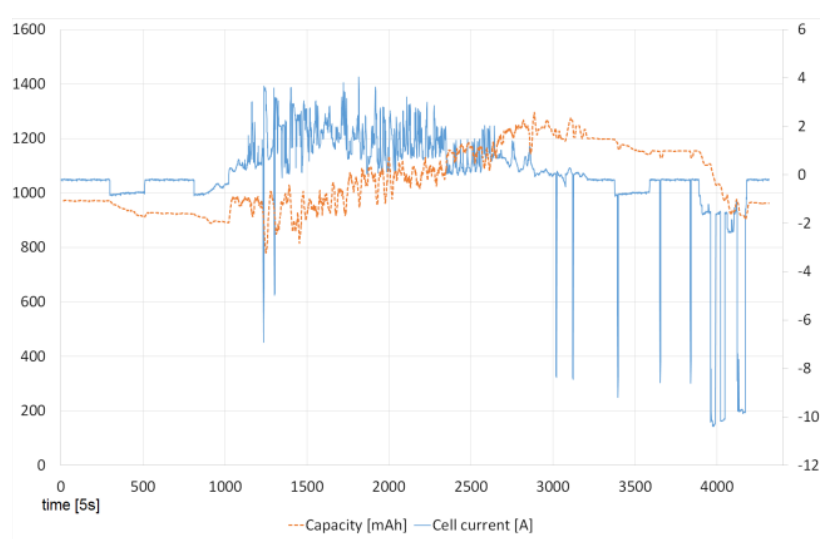

Figure 5. The dependence of available capacity as a function of time for the real data municipal type load.

One cell of the battery energy storage response to load above, generated during simulation is shown in Fig. 5 .
REP-SAIL project implements workload model based on power summing of particular energy receivers in a function of time. Workload characteristic consists of following device groups: cabin equipment, navigation and yacht positioning systems, lighting, drive and its controls, yacht controls. Additionally assumed was energy production from photovoltaic panels with total power of 1,5 kW. Result workload plot with a battery response is shown in Fig. 6.

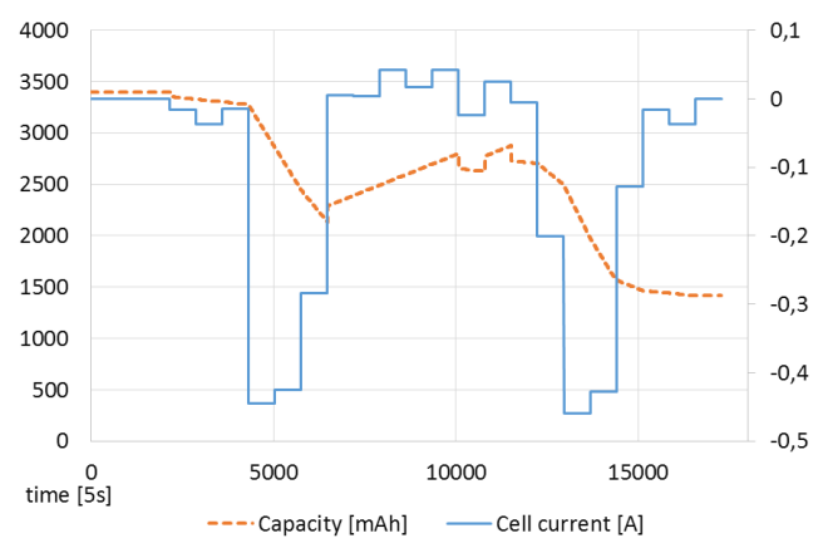

Figure 6. The dependence of available capacity as a function of time for the load created on the basis of projected energy demand for the work of REP-SAIL yacht in the motor cruise mode.

\section{Summary}

Finished analysis and acquired runs allow for observation of characteristics of battery work in given operating conditions. It's possible to simulate work of multiple types of cells based on measurement data acquired according to universal research plan. Thanks to aging process analysis for given conditions results closely reflect battery lifetime that is possible to achieve in particular application. Assembled laboratory stand will be serve to collect cells operational data in the ongoing project "Renewable Energy Powered Hybrid Innovative Sailing Yacht" implemented at the Faculty of Transport, Warsaw University of Technology. The collected data will be used to build computer models of chemical cells for the defined cycles cruise operational analysis and the types of cells selection for the battery pack cells selection.

Authors plan to develop computer model responsible for cell and battery work simulations, as well as the workplace so the test of whole battery modules may be possible.

\section{Acknowledgement}

The project is funded by the National Center for Research and Development under the ERA - NET TRANSPORT programme

\section{References}

1. M. Dubarry, N. Vuillaume, B. Y. Liaw, From Li-ion single cell model to battery pack simulation, 17th IEEE CCA, pp. 708-713 (2008). 
2. M. Dubarry, N. Vuillaume, B. Y. Liaw. From single cell model to battery pack simulation for Li-ion batteries. J. Power Sources, 186, 500-507 (2009).

3. T. GUENA, P. LEBLANC, How Depth of Discharge Affects the Cycle Life of Lithium-Metal-Polymer Batteries, INTELEC (2006).
4. J.M. Tarascon, M. Armand, Issues and challenges facing rechargeable lithium batteries, Nature 414 359-367 (2001).

5. H. Popp, J. Attia, F. Delcorso, A. Trifonova, Lifetime analysis of four different lithium ion batteries for (plug - in) electric vehicle, TRA Proceedings. (2014). 